\title{
Editorial
}

\section{Big Data and Employee Wellbeing: Walking the Tightrope between Utopia and Dystopia}

\author{
Carolyn Axtell ${ }^{1, *} \mathbb{D}$, Mark Taylor ${ }^{2} \mathbb{D}$ and Bridgette Wessels ${ }^{3}$ \\ 1 Institute of Work Psychology, Sheffield University Management School, Sheffield S10 1FL, UK \\ 2 Melbourne Law School, University of Melbourne, Parkville, VIC 3010, Australia; taylor.m@unimelb.edu.au \\ 3 School of Social \& Political Sciences, University of Glasgow, Glasgow G12 8RS, UK; \\ Bridgette.wessels@glasgow.ac.uk \\ * Correspondence: c.m.axtell@sheffield.ac.uk
}

Received: 15 November 2019; Accepted: 18 November 2019; Published: 22 November 2019

check for updates

\begin{abstract}
This special issue was inspired by an Economic \& Social Research Council funded seminar series that explored the possibilities for using Big Data and data analytics for assessing health and wellbeing risks within organisations. The aim of this special issue was to build on some of the themes developed in the seminar series and draw together and update some key insights from different disciplinary perspectives on the opportunities, challenges and lessons that could be applied in this area. This editorial, therefore, draws together the findings and themes from the submitted papers and interprets these in light of the findings from the seminar series.
\end{abstract}

Keywords: big data; employee wellbeing; multidisciplinary

This special issue was inspired by an Economic and Social Research Council (ESRC) funded seminar series (see DEW 2015-2017) that explored the possibilities for using Big Data and data analytics for assessing health and wellbeing risks within organisations. This series involved inputs from multiple disciplines across the social sciences (management, work psychology, sociology, law and information science) and resulted in the development of a set of themes and insights relating to the application of data analytics to employee wellbeing. The aim of this special issue was to build on some of the themes developed in the seminar series and draw together and update some key insights from different disciplinary perspectives on the opportunities, challenges and lessons that could be applied in this area.

A multidisciplinary perspective is required due to the complexity of issues within this area (including the technical difficulties of handling this data, concerns about what decisions or actions will be taken as a result of the analysis and their impact on employees, and issues relating to data protection, trust, and consent). Big Data is information that is large-scale (terabytes or larger), complex and varied, and which cannot be reasonably managed by traditional data management and analytical tools (Tien 2013). Organisations capture huge amounts of varied data on their employees' activities. To give an example of the amount of data available, if we consider email communication alone, a study in a large IT company with just over 30,000 employees captured 114 million dyadic email communications on their server within a four-month period (Kleinbaum et al. 2013). Already, some organisations are starting to use Big Data and data analytics to examine employee health and wellbeing, such as through the use of fitness tracking technology (The Guardian 2014) and using surveillance technology like 'Isaak' to provide real time insights to wellbeing via email and work overload monitoring (StatusToday 2019). Whilst organisations may use data responsibly and in the best interests of the employees, this is not always the case, as with the fingerprint technology used at a leisurewear company to identify and question staff who said they were 'dissatisfied' (The Guardian 2017). The motives behind using such data may be complex and the perceptions of the different stakeholders involved might vary. Therefore, there is an urgent need for input from social scientists to examine the impact of such analytics on 
organisational behavior and social systems, as well as the legal perspectives, as otherwise there is the danger that such systems could be applied in a way that does not adequately respect the employees' best interests. Moreover, opportunities in this area are likely to expand. There are vast amounts of digitally captured data gathered within organisations (e.g., network traffic, log-in/off data) that are currently under-utilized and not analysed (Priceonomics 2019), but could be used to indicate areas of highly intensive workload or other kinds of stressor where wellbeing risks might occur.

Two opposing scenarios can be identified that represent a more utopian vision and a more dystopian vision of how data might be used for monitoring employee wellbeing (Axtell 2018). The utopian vision exemplifies a scenario where there is 'co-ownership' between employee and employer relating to the collection, interpretation and use of data, existing within a supportive, caring and trusting culture, where there is a genuine concern for employee wellbeing. The dystopian vision represents a scenario where there is a lack of trust and openness, and where the initiative is driven purely by organisational needs and not the best interests of employees. These scenarios could be seen as two extremes at either end of a continuum or 'tightrope' and organisational practice may be somewhere in between these, trying to balance employee and organisational needs.

The key themes and concerns developed within the seminar series that related to these opposing visions were the importance of employee involvement in and ownership of the process, the nature of the organisational culture and its motivations, and also the skills and infrastructure required to run such an initiative. This special issue addresses these themes at three different levels: Individual, Organizational and Legal Context, and highlights the interconnection between these levels.

\section{Synthesis and Analysis of Contributions}

Calvard's contribution (Calvard 2019) outlines the psychological, sociological and critical perspectives relating to power and control when employees are involved in self-tracking their health and wellbeing. He highlights the tension between organisational needs and individual needs. For instance, whilst there might be positive motives on the organisation's part that underlay fitness tracking (e.g., relating to the better management of employee wellbeing), this can result in a tension at the individual level around privacy and surveillance of employee activity. Moreover, whilst being involved in such wellbeing programmes might seem like an exercise of an employee's own free will, organisations may incentivise participation in their own interests.

Calvard also emphasises the tension between empowerment and invasion in how data is interpreted and used by the organisation. Data that is collected is limited and only provides part of the relevant information when considering employee wellbeing, which can be interpreted in different ways by different stakeholders. How such data is interpreted could be very different depending on the organisational culture for employee involvement, so that employees may feel empowered in some organisations but invaded in others. Interpretation of data should ideally therefore involve both bottom-up (employee informed) interpretation as well as top-down (organisational level). Calvard also makes an important point about the danger of pushing the responsibility for health and wellbeing to the individual in some of these fitness tracking programmes, without appropriate organisational support and without the organisation taking enough responsibility for addressing the demands placed on employees that contribute to stress and poor wellbeing.

The contribution by Nocker and Sena (Nocker and Sena 2019) focuses on the organisational perspective and the application of Big Data analytics to talent management (talent analytics). They explore how such data can improve organisational decision making, but also discuss the costs and challenges associated with such data driven decision making. Of note in their article is the dominant emphasis on performance by organisations rather than on wellbeing per se, which contributes to the tension between organisational and employee interests. They also point out the current difficulty in linking aggregate data to performance, as there is a lack of theory and models that link individual behaviours to organisational outcomes. Nocker and Sena also emphasise the tension between access to data analytics skills and access to HR and other relevant domain knowledge for interpreting and using 
data. The data, and the skills for analysing and interpreting it, can be dispersed across multiple and different silos within organisations, which presents challenges for bringing relevant data together and using it in meaningful ways.

A core lesson from Nocker and Sena's contribution is the importance of the process of implementing these data analytics projects. Senior management support, data availability, investment in storage infrastructure and data analytics capabilities are all vital components for these projects. An organizational framework is presented for the ethical use of personal data on workers, with key stakeholders involved in talent analytics governance bodies. Within this process, they also emphasise the importance of developing trust between employees and management, and consulting with employees, not only to gain consent but to help develop programmes that are designed to benefit the employee-not just the employer.

The contribution by Taylor and Prictor (Taylor and Prictor 2019) focuses on the wider legal framework within England and Wales that drives and inhibits the use of employee data for wellbeing prediction. In particular, they emphasise the tension and competing perspectives between health and safety legislation (which emphasises the organisation's duty to assess risks to employees and remove these risks), versus data protection legislation which focuses on privacy and data governance and addresses the re-use of personal data for purposes beyond which it was originally collected. Considering the utopian and dystopian visions outlined earlier, health and safety law and data protection law may represent two approaches to addressing the misalignment of employer and employee interests. Health and safety law focuses on the organisation's 'duty of care', motivating the employer to monitor health and wellbeing, and pushes toward the utopian vision: allowing organisations to lead on good practice and go beyond existing legal duties. Data protection law emphasises the need for protection against the less benevolent motives that an organisation might have. Particularly if processing data relating to health, there are very limited exceptions to a general prohibition of processing. None of these exceptions seems an easy fit with an organisation going beyond existing legal duties to use data in creative ways to improve the practice/risk relationship for employees.

Taylor and Prictor highlight that, with advances in technology and data analytics, the ability to identify relevant associations between measurable workplace activities and risks to wellbeing is increasingly becoming a reality. Importantly, they suggest that if such methods become more commonplace, then organisations who are not monitoring employees for wellbeing risks using such methods could be considered as failing in their duty of care. On the other hand, data protection law may not currently encourage the kinds of data processing that could come to be expected of responsible employers (i.e., to examine the risks associated with their workplace practices). Further, they suggest that the restrictions placed within data protection law could hamper progress towards understanding the relationship between organisational practices and wellbeing, unless it becomes a legal requirement to monitor employees in this way.

Taylor and Prictor's contribution highlights the importance of the organisation's motives for monitoring employees. They also point to the importance of employee involvement and consultation in any projects where data is used to assess risks to employees. These issues go beyond 'consent' and towards the co-design and co-evaluation of any such projects so that employees see these initiatives as fair and proportionate.

All the articles in the special issue identify the tensions and the paradoxical and competing perspectives around using Big Data for the benefit of employee health and wellbeing. Whilst there could be opportunities to do great good with the data, paradoxically, at the same time there could be potential for harm. The outcomes may neither be entirely good nor entirely bad. Perhaps this is what needs to be recognised and understood by employees, organisations and social scientists alike. It may be easy to be disheartened by these apparent tensions, which invite action on the one hand but at the same time deter action. However, these tensions and competing perspectives are beneficial and serve a purpose. A tightrope cannot be traversed unless there is tension. The utopian and dystopian perspectives may need to exist in close proximity so that nothing is taken for granted, checks are made, opposing viewpoints are considered and we tread very carefully, with balance. The findings from this 
special issue highlight the interdependence between the different opposing perspectives and our need to accept these tensions and work with them.

Central to balancing these tensions is employee involvement and ownership of relevant processes. All the articles in the special issue have highlighted that just relying on consent is not enough, especially when there are power imbalances between employee and employer. Employees need to be involved in the design and development of any data analytics initiatives that involve data and decisions about them. There needs to be careful co-design between employee and employer when considering data collection (what will be collected and how), in the interpretation of findings and how that data will be used (e.g., when designing any interventions that occur as a result). The underlying organisational motives must be clear, and the interests of employee and organisation need to be understood, considered and balanced, in any attempts to monitor employee wellbeing.

If it eventually becomes a requirement for organisations to try and predict wellbeing problems using the data available to them, then organisations will need to know how to manage such projects and negotiate the minefield of different interests, perspectives and legal frameworks without falling off the tightrope. Therefore, this special issue has highlighted that organisations should not just be investing in building talent analytics skills, but also investing in developing their processes for employee involvement and co-design, so that they are able to effectively involve employees in the design and management of these projects. Employee trust in the future will be dependent on them perceiving data use to be a reasonable expectation.

\section{Conclusions}

This special issue has confirmed and extended the findings from the seminar series in two important ways. First, it has highlighted that the tensions and opposing scenarios within this area exist interdependently, with the potential for good and bad outcomes occurring simultaneously. Further research could help enhance understanding of how organisations can balance these competing perspectives within their data analytics projects. Second, this special issue has highlighted the need for organisations to invest in employee involvement and co-design expertise. The ability to involve employees and other relevant stakeholders in these processes is likely to be as important as the data analytics expertise required to make such projects a reality. Future research could examine how these co-design processes might be facilitated within organisations, and what works to build trust in different types of organisation with different cultures and structures. We hope these insights from the special issue will inspire new lines of enquiry relating to the use of Big Data and data analytics for enhancing employee wellbeing.

Funding: The seminar series that inspired this special issue was funded by the Economic and Social Research Council (ESRC). Grant title: Exploring Big Data to Examine Employee Health and Wellbeing: A Seminar Series, grant reference: ES/N00891X/1.

Acknowledgments: Our co-editor Christine Sprigg sadly passed away after a long illness before this editorial was written and the special issue completed. However, we would like to acknowledge the contribution that Christine made to the ideas that were included in the call for this special issue, the editorial and the seminar series that proceeded it. We therefore dedicate this editorial in memory of her.

Conflicts of Interest: The authors declare no conflict of interest. The funders had no role in the special issue or editorial.

\section{References}

Axtell, Carolyn. 2018. Big Data Could Bring about Workplace Utopia-Or the Office from Hell. January 4. Available online: https://theconversation.com/big-data-could-bring-about-workplace-utopia-or-the-officefrom-hell-86967 (accessed on 5 January 2018).

Calvard, Thomas. 2019. Integrating Social Scientific Perspectives on the Quantified Employee Self. Social Sciences 8: 262. [CrossRef] 
DEW. 2015-2017. Data and Employee Wellbeing: An Introduction to the Big Data and Employee Wellbeing Seminars. Available online: http://www.dew.group.shef.ac.uk/ (accessed on 12 November 2019).

Kleinbaum, Adam M., Toby E. Stuart, and Michael L. Tushman. 2013. Discretion without constraint: Homophily and structure in a formal organization. Organization Science 24: 1316-36. [CrossRef]

Nocker, Manuela, and Vania Sena. 2019. Big Data and Human Resources Management: The Rise of Talent Analytics. Social Sciences 8: 273. [CrossRef]

Priceonomics. 2019. Companies Collect a Lot of Data But How Much Do They Actually Use? Available online: https://priceonomics.com/companies-collect-a-lot-of-data-but-how-much-do/ (accessed on 23 October 2019).

Taylor, Mark J., and Megan Prictor. 2019. Insight or Intrusion? Correlating Routinely Collected Employee Data with Health Risk. Social Sciences 8: 291. [CrossRef]

The Guardian. 2014. These Companies Are Tracking the Fitness of Their Employees. Available online: https:/www.theguardian.com/technology/2014/mar/17/why-companies-are-tracking-the-fitnessof-their-employees (accessed on 23 October 2019).

The Guardian. 2017. Sports Direct Workers Invited to Press Sad or Happy Emoji Clocking In. Available online: https://www.theguardian.com/business/2017/sep/01/sports-direct-warehouse-staff-sad-happy-emojifingerprint-survey-workplace (accessed on 13 November 2019).

StatusToday. 2019. Available online: https://www.statustoday.com (accessed on 26 August 2019).

Tien, James M. 2013. Big data: Unleashing information. Journal of Systems Science and Systems Engineering 22: 127-51. [CrossRef]

(C) 2019 by the authors. Licensee MDPI, Basel, Switzerland. This article is an open access article distributed under the terms and conditions of the Creative Commons Attribution (CC BY) license (http://creativecommons.org/licenses/by/4.0/). 